\title{
Diagnostic Performance of a Smart Device With Photoplethysmography Technology for Atrial Fibrillation Detection: Pilot Study (Pre-mAFA II Registry)
}

Yong-Yan Fan ${ }^{1,2}$, PhD; Yan-Guang Li ${ }^{2}$, PhD; Jian $\mathrm{Li}^{2}$, PhD; Wen-Kun Cheng ${ }^{2}$, MS; Zhao-Liang Shan ${ }^{2}, \mathrm{MD}, \mathrm{PhD}$; Yu-Tang Wang ${ }^{1,3}, \mathrm{MD}, \mathrm{PhD}$; Yu-Tao Guo ${ }^{2}, \mathrm{MD}, \mathrm{PhD}$

${ }^{1}$ College of Medicine, Nankai University, Tianjin, China

${ }^{2}$ Department of Cardiology, Chinese People's Liberation Army General Hospital, Beijing, China

${ }^{3}$ Department of Geriatric Cardiology, Chinese People's Liberation Army General Hospital, Beijing, China

\section{Corresponding Author:}

Yu-Tao Guo, MD, PhD

Department of Cardiology

Chinese People's Liberation Army General Hospital

28 Fuxing Rd

Beijing, 100853

China

Phone: 8613810021492

Email: guoyutao2010@126.com

\section{Abstract}

Background: Atrial fibrillation (AF) is the most common sustained cardiac arrhythmia. The asymptomatic nature and paroxysmal frequency of AF lead to suboptimal early detection. A novel technology, photoplethysmography (PPG), has been developed for AF screening. However, there has been limited validation of mobile phone and smart band apps with PPG compared to 12-lead electrocardiograms (ECG).

Objective: We investigated the feasibility and accuracy of a mobile phone and smart band for AF detection using pulse data measured by PPG.

Methods: A total of 112 consecutive inpatients were recruited from the Chinese PLA General Hospital from March 15 to April 1, 2018. Participants were simultaneously tested with mobile phones (HUAWEI Mate 9, HUAWEI Honor 7X), smart bands (HUAWEI Band 2), and 12-lead ECG for 3 minutes.

Results: In all, 108 patients (56 with normal sinus rhythm, 52 with persistent AF) were enrolled in the final analysis after excluding four patients with unclear cardiac rhythms. The corresponding sensitivity and specificity of the smart band PPG were 95.36\% (95\% CI 92.00\%-97.40\%) and 99.70\% (95\% CI 98.08\%-99.98\%), respectively. The positive predictive value of the smart band PPG was 99.63\% (95\% CI 97.61\%-99.98\%), the negative predictive value was 96.24\% (95\% CI 93.50\%-97.90\%), and the accuracy was $97.72 \%$ (95\% CI 96.11\%-98.70\%). Moreover, the diagnostic sensitivity, specificity, positive predictive value, negative predictive value, and accuracy of mobile phones with PPG for AF detection were over 94\%. There was no significant difference after further statistical analysis of the results from the different smart devices compared with the gold-standard ECG (P>.99).

Conclusions: The algorithm based on mobile phones and smart bands with PPG demonstrated good performance in detecting $\mathrm{AF}$ and may represent a convenient tool for $\mathrm{AF}$ detection in at-risk individuals, allowing widespread screening of $\mathrm{AF}$ in the population.

Trial Registration: Chinese Clinical Trial Registry ChiCTR-OOC-17014138; http://www.chictr.org.cn/showproj.aspx?proj=24191 (Archived by WebCite at http://www.webcitation/76WXknvE6)

(JMIR Mhealth Uhealth 2019;7(3):e11437) doi: 10.2196/11437

\section{KEYWORDS}

atrial fibrillation; photoplethysmography; detection; accuracy; mobile phone; smart band; algorithm 


\section{Introduction}

Atrial fibrillation (AF) is the most common sustained cardiac arrhythmia encountered in clinical practice and is associated with increased risk of stroke, systemic embolism, heart failure, hospitalization, and death [1-3]. At least one-third of patients with AF are asymptomatic [4]. Due to the asymptomatic nature and paroxysmal frequency of this arrhythmia, early detection is challenging and often unsuccessful [5]. Strikingly, acute stroke or heart failure is often the first sign of AF [6,7]. Asymptomatic AF is also related with worse outcomes compared with symptomatic AF [8]. However, approximately $70 \%$ of AF-related strokes could be avoided with early detection and early management, such as initiation of oral anticoagulants [9]. The European Society of Cardiology now recommends screening of AF in the primary care of high-risk patients, including those aged 65 years and older, which is important for the prevention of stroke [10].

Although studies have shown that more frequent monitoring can improve AF detection [11-13], population-based screening remains suboptimal because of the inconvenience and high expense of current screening approaches, such as Holter monitoring with different time durations ( 24 hours and 7 days) $[14,15]$. In addition, the diagnostic value of the standard 12-lead electrocardiogram (ECG) is limited by the need for patients to obtain ECG diagnostic equipment, as well as the requirement that AF be present at the time of the ECG recording. As such, we need a more convenient heart rhythm screening or detection approach to identify AF and initiate early treatment.

Technical options to detect AF have significantly improved within the past decade. Different technologies such as blood pressure monitors [16], single-lead cardiac event monitors (Kardia Mobile case or card) [17], and mobile phone apps using photoplethysmographic signals have emerged for this purpose $[18,19]$. Taggar et al [20] analyzed 21 studies that investigated 39 interventions for detecting AF before March 16, 2015. Their meta-analysis found that the most accurate methods for detecting AF were blood pressure monitors, which had a sensitivity of $98 \%$ and a specificity of $92 \%$, and non-12-lead ECG, which had a sensitivity of $91 \%$ and a specificity of $95 \%$. Although blood pressure monitors and non-12-lead ECG both had relatively high diagnostic accuracies, the need for a blood pressure monitor and a clinical specialist to analyze the non-12-lead ECG pose a challenge for wide-scale implementation of AF screening. ECG hand-held devices usually provided a single-lead ECG for detecting AF, which including two different types: on-device diagnostic algorithm and transmitted data devices. According to the data from the sixth Atrial Fibrillation NETwork (AFNET) and the European Heart Rhythm Association (EHRA) Consensus Conference [21], the sensitivity of an on-device diagnostic algorithm was $94 \%$ to $98 \%$, and the specificity was $76 \%$ to $97 \%$. Transmitted data devices achieved a sensitivity and specificity of $94 \%$ to $96 \%$ and $90 \%$ to $95 \%$ for AF detection, respectively [21]. However, the diagnostic sensitivity of photoplethysmography (PPG) for AF detection was $97 \%$ to $100 \%$, and the specificity was $92 \%$ to $94 \%$ [21]. The potential of PPG technology for AF detection has been confirmed in some small sample studies, but it is still necessary to verify the diagnostic accuracy of PPG technology for AF detection with a larger sample size study in the real world.

Available studies have indicated that PPG technology is a promising technology for AF screening. PPG is an optical method measuring changes in tissue blood volume through the skin capillary bed, which can be performed by using a mobile phone without any additional peripheral equipment [22,23]. The PPG waveform is generally acquired by the built-in camera of a mobile phone to measure pulsatile changes in light absorption reflected from a fingertip illuminated by the light-emitting diode (LED) flash [19]. Most smart bands currently on the market use PPG technology, and heart rate sensors on most smart bands work via PPG [24]. Detecting AF using easily accessible devices, such as mobile phones and smart bands, may represent a novel opportunity to passively and automatically detect asymptomatic AF that does not require additional hardware and is simple to operate. However, there has been limited validation of mobile phone and smart band PPG compared to 12-lead ECG, and the consistency and stability of PPG technology in AF screening with different smart devices still lack accurate data.

In this study, we tested the hypothesis that pulse waveform signals recorded using smart devices, including mobile phones and smart bands, can be analyzed by a realizable algorithm and can distinguish AF from normal sinus rhythm. This study also provides data on AF screening technology for the Chinese population.

\section{Methods}

\section{Study Population}

A total of 112 consecutive inpatients were recruited from the Chinese People's Liberation Army General Hospital (Beijing, China) from March 15 to April 1, 2018. Information regarding demographic characteristics, medical history, blood test results, and medications were recorded.

Patients aged 18 years and older were included in the study. Exclusion criteria included patients unable to use mobile phones and smart bands, with mental or memory problems, or with a pacemaker or implantable cardioverter defibrillator. Written informed consent was obtained and signed by each individual willing to take part in the study.

The mobile atrial fibrillation apps (mAFA) II registry is mobile health (mHealth) technology for improved screening, patient involvement, and optimizing integrated care of AF. Ours was a single-center pilot study of AF screening that was pre-mAFA II registry. The Medical Ethics Committee of the Chinese PLA General Hospital and the China Food and Drug Administration approved the study protocol (no: S2017-105-02). The study is registered in the Chinese Clinical Trial Registry, International Clinical Trials Registry Platform of the World Health Organization (ChiCTR-OOC-17014138).

\section{Signal Acquisition and Processing}

Mobile phones (HUAWEI Mate 9, HUAWEI Honor 7X; Huawei Technologies Co, Ltd, Shenzhen, China) and smart bands 
(HUAWEI Band 2) were used for collecting pulse waveform signals. Pulse waveform recordings were performed by the participants under the supervision of trained study personnel. A dedicated data collection app, Heartbeats (Preventicus GmbH, Jena, Germany), was responsible for the pulse waveform signal acquisition and was installed in the HUAWEI mobile phones.

Participants were simultaneously tested with mobile phones (HUAWEI Mate 9, HUAWEI Honor 7X), smart bands (HUAWEI Band 2), and 12-lead ECG for 3 minutes. Participants were advised to lie down in a supine position and breathe spontaneously. A HUAWEI Mate 9 (mobile phone 1) was positioned on the left-hand finger (either the index or middle finger) with the camera lens and LED light placed on the fingertip of the participant. Similarly, a HUAWEI Honor 7X (mobile phone 2) was positioned on the finger of the right hand. PPG measurements were performed by using the Heartbeats mobile phone app. The participant was asked to wear two smart bands, one each on the left and right hand. A 3-minute pulse waveform recording was obtained from each participant using the smart devices and a formal 12-lead ECG simultaneously. The PRO AF PPG was not running on the mobile devices in real time but remotely in the cloud. Then all 3-minute pulse waveform recordings using the smart devices were uploaded to the online cloud center and analyzed by a realizable algorithm (PRO AF PPG) provided by Preventicus (Preventicus GmbH, Jena, Germany). Figure 1 shows a prototype for AF detection using HUAWEI mobile phones and smart bands.

Figure 1. A prototype for atrial fibrillation detection using HUAWEI mobile phones and smart bands. A: A patient is simultaneously checked with HUAWEI mobile phones (Mate 9, Honor 7X), HUAWEI smart bands (Band 2), and 12-lead ECG. B: A fingertip is placed in contact with the built-in camera lens of a HUAWEI Mate 9 mobile phone and is illuminated by the adjacent LED flash. C: A screenshot of the pulse waveform data collection app (Heartbeats) running on a HUAWEI Mate 9 mobile phone. D: Representative pulse waveform recording from a patient with normal sinus rhythm. E: Representative pulse waveform recording from a patient with persistent atrial fibrillation.
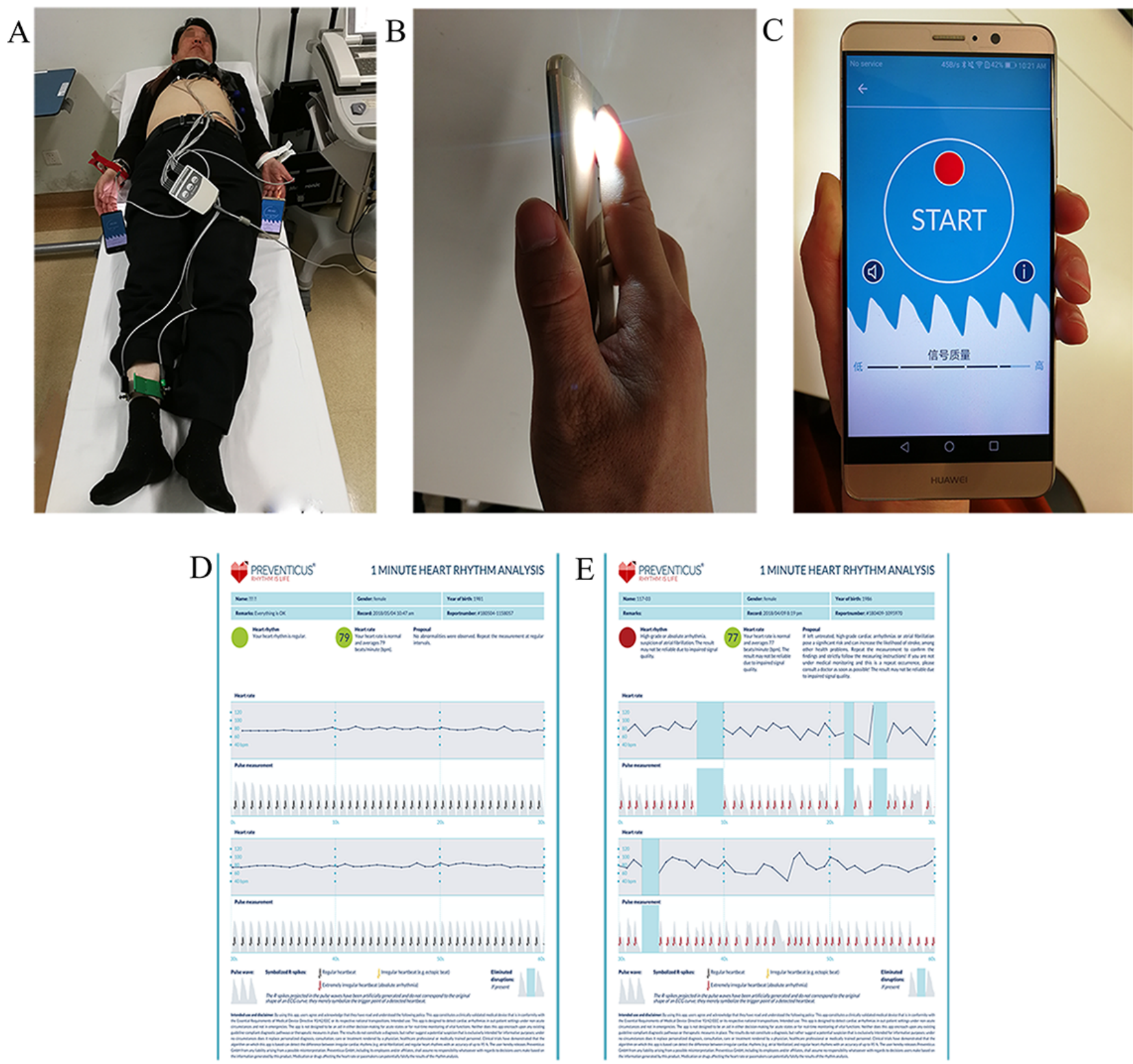


\section{Rhythm Diagnosis}

Results from an ECG remain the gold standard for the measurement of heart rhythms, and they were confirmed by two independent cardiologists who were blinded to the baseline information of participants. The results of the algorithm were independently reviewed for each 12-lead ECG. For participants whose ECGs were initially affected by artifacts, trained study personnel instructed them to repeat the recordings to provide an optimal tracing for subsequent reading by the cardiologists. The Heartbeats app added a pulse waveform quality assessment step to reject recordings that were corrupted or too noisy and to prompt the user to retake a measurement.

After data collection, the novel heartbeat detection algorithm (PRO AF PPG), based on a combination of morphology and frequency analysis of the pulse waveform, was applied to perform beat-to-beat rhythm analysis and determine whether or not the participant suffered from AF. The diagnostic performance of the algorithm in detecting AF was then evaluated using the 12-lead ECG interpretation as the standard. Figure 2 shows a flowchart of the study.

\section{Statistical Analysis}

Continuous variables were tested for normality by the Kolmogorov-Smirnov test. Data with a normal distribution are presented as means and standard deviations and were analyzed using the $t$ test for two independent samples. Data with a nonnormal distribution are presented as medians and interquartile ranges (IQRs) and were analyzed using the Mann-Whitney $U$ test. Data with discrete variables are presented as percentages and were analyzed using the Pearson chi-square test or Fisher exact test.

Figure 2. A flowchart of the study. AF: atrial fibrillation; ECG: electrocardiogram; SR: sinus rhythm.

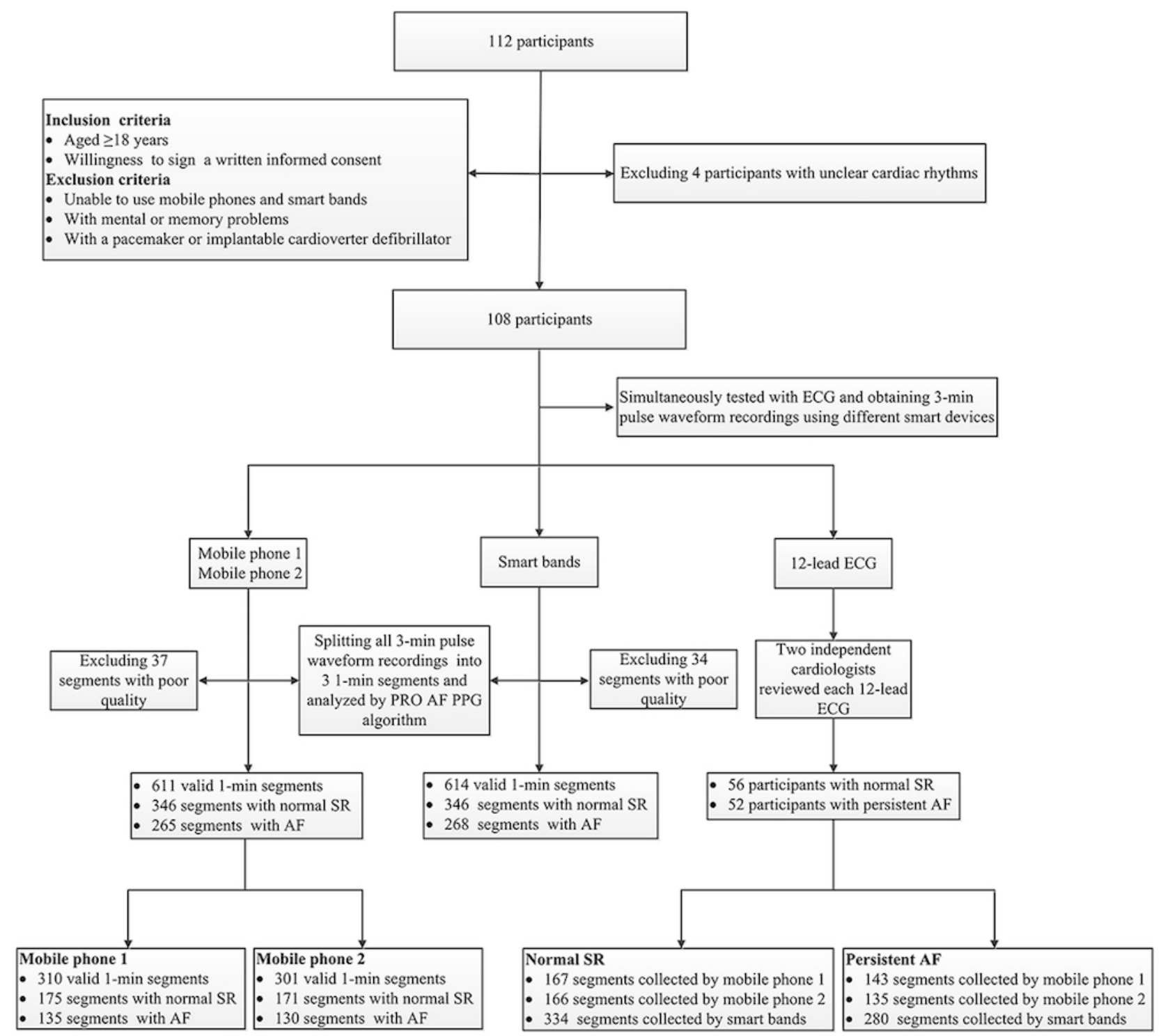


Sensitivity, specificity, positive predictive value, negative predictive value, and accuracy with $95 \% \mathrm{CI}$ were used to measure the performance of our AF screening algorithm in the smart devices. The diagnostic performance of the algorithm in different devices was evaluated against reference ECG recordings, from which was calculated the number of true positives (TP), true negatives (TN), false positives (FP), and false negatives (FN). Sensitivity, specificity, positive predictive value, negative predictive value, and accuracy for AF diagnosis were calculated as simple proportions for the PRO AF PPG algorithm. The sensitivity was calculated as $\mathrm{TP} /(\mathrm{TP}+\mathrm{FN}$ ) (true positives divided by all positives) and specificity as $1-\mathrm{FP} /(\mathrm{TN}+\mathrm{FP})$ (true negatives divided by all negatives). The corresponding positive predictive value was defined as $\mathrm{TP} /(\mathrm{TP}+\mathrm{FP})$, and the negative predictive value as $\mathrm{TN} /(\mathrm{FN}+\mathrm{TN})$. The corresponding accuracy was calculated as $(\mathrm{TP}+\mathrm{TN}) /(\mathrm{TP}+\mathrm{TN}+\mathrm{FP}+\mathrm{FN})$. Statistical evaluation was performed with SPSS 19.0 (SPSS Inc, Chicago, IL, USA). A value of $P<.05$ was considered statistically significant.

\section{Results}

Among the 112 participants who fulfilled the inclusion criteria for the study, four participants were excluded because the ECG recordings showed unclear cardiac rhythms. As a result, 108 patients (56 with normal sinus rhythm, 52 with persistent AF) were enrolled in the final analysis. Table 1 summarizes the clinical characteristics of the study population. Participants with persistent AF were significantly older $(P=.002)$, had a higher body mass index $(P=.02)$, and had more prevalent heart failure $(P=.006)$. Thromboembolic risk and bleeding risk were higher in participants with persistent $\mathrm{AF}$ compared to those with normal sinus rhythm based on $\mathrm{CHA}_{2} \mathrm{DS}_{2}$-VASc score (median 3, IQR 2-5 vs median 2, IQR 1.3-3.75, $P=.003$ ) and HAS-BLED score (median 2, IQR 1-2 vs median 1, IQR 0-2, $P=.005$ ), respectively. The use of oral anticoagulants for preventing stroke was $77 \%$ (40/52) in participants with persistent $\mathrm{AF}$ and $18 \%(10 / 56)$ in participants with normal sinus rhythm $(P<.001)$. The use of diuretics and digoxin were significantly higher in participants with persistent AF compared to those with normal sinus rhythm ( $P=.03$ and $P=.02$, respectively), as well as the use of class III antiarrhythmic drugs $(P<.001)$.

We split the 3-minute pulse waveform recordings of each participant obtained from mobile phones and smart bands into three 1-minute segments for further analysis with results from the 12-lead ECG. After splitting, there were 614 valid 1-minute segments of pulse waveform recordings in total obtained from smart bands, divided into 280 for AF and 334 for normal sinus rhythm based on ECG. Thirty-four 1-minute segments of signal recordings were deemed poor quality and were disregarded. The diagnostic performance of the PRO AF PPG AF screening algorithm in smart bands was evaluated against reference ECG recordings and demonstrated a sensitivity of $95.36 \%(95 \% \mathrm{CI}$ $92.00 \%-97.40 \%)$ and a specificity of $99.70 \%(95 \%$ CI 98.08\%-99.98\%) for the detection of AF. The corresponding positive predictive value of the PRO AF PPG algorithm for AF screening was $99.63 \%$ (95\% CI 97.61\%-99.98\%), the negative predictive value was $96.24 \%$ (95\% CI $93.50 \%-97.90 \%)$, and the accuracy was $97.72 \%$ (95\% CI 96.11\%-98.70\%).

For mobile phones, we obtained 611 valid 1-minute segments of pulse waveform recordings in total, of which 310 were obtained from mobile phone 1 and 301 from mobile phone 2, divided into 278 for AF and 333 for normal sinus rhythm based on standard ECG recordings. Thirty-seven 1-minute segments of signal recordings were omitted because of poor quality, of which 14 were recorded by mobile phone 1 and 23 by mobile phone 2 . The diagnostic sensitivity and specificity of the PRO AF PPG algorithm for AF detection using mobile phone 1 and mobile phone 2 were $94.96 \%$ (95\% CI $91.51 \%-97.11 \%$ ) and $99.70 \%$ (95\% CI $98.07 \%-99.98 \%$ ), respectively. The positive predictive value was $99.62 \%$ (95\% CI $97.59 \%-99.98 \%)$, the negative predictive value was $95.95 \% \quad(95 \%$ CI 93.15\%-97.68\%), and the accuracy of the algorithm for $\mathrm{AF}$ detection was $97.55 \%$ (95\% CI\% 95.89\%-98.57\%). Moreover, the diagnostic sensitivity, specificity, positive predictive value, negative predictive value, and accuracy of mobile phone 1 alone or mobile phone 2 alone with PPG for AF detection were over 94\%. Detailed diagnostic performance of the PPG technology for AF screening in different smart devices is summarized in Table 2. There was no significant difference in further statistical analysis of the results from different smart devices compared with ECG $(P>.99)$.

Table 3 lists data from the literature on AF detection with different technologies. Compared with recent studies on AF detection, the PRO AF PPG algorithm showed good diagnostic performance from each smart device. 
Table 1. Baseline characteristics of participants $(\mathrm{N}=108)$.

\begin{tabular}{|c|c|c|c|}
\hline Characteristics & Sinus rhythm $(\mathrm{n}=56)$ & Persistent $\mathrm{AF}^{\mathrm{a}}(\mathrm{n}=52)$ & $P$ value \\
\hline \multicolumn{4}{|l|}{ Demographics } \\
\hline Age (years), mean (SD) & $58(14.78)$ & $66.56(13.17)$ & .002 \\
\hline Female, n $(\%)$ & $26(46)$ & $19(37)$ & .30 \\
\hline Body mass index $\left(\mathrm{kg} / \mathrm{m}^{2}\right)$, mean (SD) & $24.44(2.88)$ & $25.98(3.97)$ & .02 \\
\hline \multicolumn{4}{|l|}{ Medical history } \\
\hline Heart failure, n $(\%)$ & $2(4)$ & $12(23)$ & .006 \\
\hline Hypertension, n (\%) & $29(52)$ & $35(67)$ & .10 \\
\hline Diabetes mellitus, n (\%) & $15(27)$ & $17(33)$ & .50 \\
\hline Previous stroke/SE ${ }^{\mathrm{b}} /$ TIA $^{\mathrm{c}}, \mathrm{n}(\%)$ & $4(7)$ & $9(17)$ & .19 \\
\hline Coronary artery disease, $\mathrm{n}(\%)$ & $25(45)$ & $19(37)$ & .39 \\
\hline Vascular disease, $\mathrm{n}(\%)$ & $31(55)$ & $37(71)$ & .09 \\
\hline $\mathrm{COPD}^{\mathrm{d}}, \mathrm{n}(\%)$ & $1(2)$ & $3(6)$ & .56 \\
\hline Renal dysfunction, n (\%) & $2(4)$ & $8(15)$ & .07 \\
\hline Hepatic dysfunction, n (\%) & 0 & $2(4)$ & .23 \\
\hline Sleep apnea, n (\%) & $2(4)$ & $6(12)$ & .22 \\
\hline Hyperthyroidism, n (\%) & $1(2)$ & $4(8)$ & .32 \\
\hline Current smoking, n (\%) & $16(29)$ & $17(33)$ & .64 \\
\hline Current drinking, $\mathrm{n}(\%)$ & $13(21)$ & $11(23)$ & .80 \\
\hline $\mathrm{CHA}_{2} \mathrm{DS}_{2}-\mathrm{VASc}^{\mathrm{e}}$ score, median $\left(\mathrm{IQR}^{\mathrm{f}}\right)$ & $2(1-3.75)$ & $3(2-5)$ & .003 \\
\hline HAS-BLED ${ }^{\mathrm{g}}$ score, median (IQR) & $1(0-2)$ & $2(1-2)$ & .005 \\
\hline \multicolumn{4}{|l|}{ Medications, n (\%) } \\
\hline Oral anticoagulant & $10(18)$ & $40(77)$ & $<.001$ \\
\hline Antiplatelet drug & $15(27)$ & $23(44)$ & .06 \\
\hline Calcium channel blockers & $17(30)$ & $13(25)$ & .54 \\
\hline $\mathrm{ACEI} / \mathrm{ARB}^{\mathrm{h}}$ & $21(38)$ & $16(31)$ & .46 \\
\hline Diuretic & $5(9)$ & $13(25)$ & .03 \\
\hline Digoxin & $3(5)$ & $11(21)$ & .02 \\
\hline \multicolumn{4}{|l|}{ Antiarrhythmic drug, n (\%) } \\
\hline Class I & $6(11)$ & $2(4)$ & .32 \\
\hline Beta blocker & $27(48)$ & $34(65)$ & .07 \\
\hline Class III & $3(5)$ & $20(38)$ & $<.001$ \\
\hline Class IV & $3(5)$ & $3(6)$ & $>.99$ \\
\hline
\end{tabular}

${ }^{\mathrm{a}} \mathrm{AF}$ : atrial fibrillation.

${ }^{\mathrm{b}} \mathrm{SE}$ : systemic arterial embolism.

${ }^{\mathrm{c}}$ TIA: transient ischemic attack.

${ }^{\mathrm{d}}$ COPD: chronic obstructive pulmonary disease.

${ }^{\mathrm{e}} \mathrm{CHA}_{2} \mathrm{DS}_{2}$-VASc: congestive heart failure, hypertension, age $\geq 75$ years, diabetes mellitus, stroke (doubled), vascular disease, age 65-74, female sex. ${ }^{\mathrm{f}} \mathrm{IQR}$ : interquartile range.

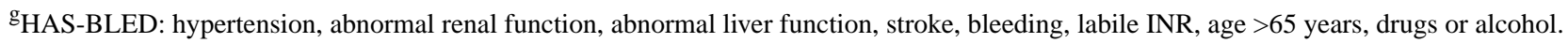

${ }^{\mathrm{h}} \mathrm{ACEI} / \mathrm{ARB}$ : angiotensin-converting-enzyme inhibitor, angiotensin receptor blockers. 
Table 2. Detailed diagnostic performance of the photoplethysmography technology for atrial fibrillation screening in different smart devices.

\begin{tabular}{llll}
\hline Index & Smart bands & Mobile phones & Mobile phone 1 \\
\hline Sensitivity, \% (95\% CI) & $95.36(92.00-97.40)$ & $94.96(91.51-97.11)$ & $94.41(88.91-97.38)$ \\
Specificity, \% (95\% CI) & $99.70(98.08-99.98)$ & $99.70(98.07-99.98)$ & $100(97.20-100)$ \\
PPV $^{\mathrm{a}}, \%(95 \% \mathrm{CI})$ & $99.63(97.61-99.98)$ & $99.62(97.59-99.98)$ & $100(96.55-100)$ \\
NPV $^{\mathrm{b}}, \%(95 \% \mathrm{CI})$ & $96.24(93.50-97.90)$ & $95.95(93.15-97.68)$ & $95.43(90.88-97.86)$ \\
Accuracy, \% (95\% CI) & $97.72(96.11-98.70)$ & $97.55(95.89-98.57)$ & $97.42(94.78-98.80)$ \\
\hline
\end{tabular}

${ }^{\mathrm{a}} \mathrm{PPV}$ : positive predictive value.

bPV: negative predictive value.

Table 3. Data from the literature on atrial fibrillation (AF) detection with different technologies.

\begin{tabular}{|c|c|c|c|c|c|}
\hline Study, year, and population studied & AF detection protocol & Sensitivity, $\%$ & Specificity, \% & $\mathrm{PPV}^{\mathrm{a}}, \%$ & $\mathrm{NPV}^{\mathrm{b}}, \%$ \\
\hline \multicolumn{6}{|l|}{ McManuset al, 2013 [25] } \\
\hline $\begin{array}{l}76 \text { patients before and after car- } \\
\text { dioversion }\end{array}$ & $\begin{array}{l}\text { An iPhone } 4 S \text {, an algorithm combining } \\
\text { RMSSD }^{c} \text { and } \mathrm{ShE}^{\mathrm{d}}\end{array}$ & 96.2 & 97.5 & $\underline{e}^{\mathrm{e}}$ & - \\
\hline \multicolumn{6}{|l|}{ Chan et al, 2016 [22] } \\
\hline 1013 patients & AliveCor automated algorithm & 71.4 & 99.4 & 76.9 & 99.2 \\
\hline \multicolumn{6}{|l|}{ Krivoshei et al, 2017 [19] } \\
\hline 80 consecutive patients & $\begin{array}{l}\text { An iPhone } 4 S \text {, an algorithm combining } \\
\text { RMSSD and ShE }\end{array}$ & 80 & 95 & - & - \\
\hline 80 consecutive patients & $\begin{array}{l}\text { An iPhone } 4 \mathrm{~S} \text {, an algorithm combining } \\
\text { RMSSD and PPA }^{\mathrm{f}}\end{array}$ & 95 & 95 & - & - \\
\hline 80 consecutive patients & $\begin{array}{l}\text { An iPhone } 4 S \text {, an algorithm combining } \\
\text { ShE and PPA }\end{array}$ & 50 & 95 & - & - \\
\hline \multicolumn{6}{|l|}{ Rozen et al, 2018 [26] } \\
\hline $\begin{array}{l}97 \text { patients before and after electri- } \\
\text { cal cardioversion }\end{array}$ & An iPhone, Cardiio Rhythm mobile app & 93.1 & 90.9 & 92.2 & 92.0 \\
\hline \multicolumn{6}{|l|}{ Bumgarner et al, 2018 [27] } \\
\hline $\begin{array}{l}100 \text { patients before and after car- } \\
\text { dioversion }\end{array}$ & $\begin{array}{l}\text { Kardia Band from AliveCor paired with } \\
\text { an Apple Smartwatch, AliveCor automat- } \\
\text { ed algorithm }\end{array}$ & 93 & 84 & - & - \\
\hline \multicolumn{6}{|l|}{ Tisonet al, 2018 [28] } \\
\hline $\begin{array}{l}51 \text { sedentary participants undergo- } \\
\text { ing cardioversion }\end{array}$ & $\begin{array}{l}\text { Smartwatch } \mathrm{PPG}^{\mathrm{g}} \text { coupled with a deep } \\
\text { neural network }\end{array}$ & 98 & 90.2 & 90.9 & 97.8 \\
\hline 1617 ambulatory participants & $\begin{array}{l}\text { Smartwatch PPG coupled with a deep } \\
\text { neural network }\end{array}$ & 67.7 & 67.6 & 7.9 & 98.1 \\
\hline
\end{tabular}

${ }^{a}$ NPV: negative predictive value.

${ }^{\mathrm{b}} \mathrm{PPV}$ : positive predictive value.

${ }^{\mathrm{c}} \mathrm{RMSSD}$ : root mean square of successive difference of RR intervals.

${ }^{\mathrm{d}} \mathrm{ShE}=$ Shannon entropy.

e Missing data.

fPPA: Poincaré plot analysis.

gPP: photoplethysmography. 


\section{Discussion}

\section{Principal Findings}

In this study, we demonstrated good diagnostic performance of the smart devices for AF detection using pulse waveform data measured by PPG. To the best of our knowledge, this is the first study on AF screening technology in a Chinese population. The main findings were (1) the PRO AF PPG algorithm demonstrated promising potential for accurate detection and discrimination of AF from normal sinus rhythm in a trial setting and may be applied to any mobile phone or smart band for AF screening, and (2) there was no significant difference in the results from different smart devices compared with ECG, and the tested models of mobile phone had no impact on the diagnostic performance of the algorithm.

In this study, HUAWEI mobile phones and smart bands showed higher consistency and stability of PPG technology in AF screening with PRO AF PPG algorithm and performed better with higher sensitivity and specificity. Prior to the development of the PRO AF PPG algorithm, several algorithms were validated for the detection of AF based on mobile phones and wearable devices (Table 3). Root mean square of successive difference of RR intervals (RMSSD), Shannon entropy (ShE), Poincaré plot analysis, and the AliveCor automated algorithm have been used to discriminate between $\mathrm{AF}$ and sinus rhythm by analyzing pulse waveform signals recorded using smart devices in several recent studies [19,22,25,27]. McManus et al [25] described an app using a camera and LED light of an iPhone $4 \mathrm{~S}$ to record pulse waves obtained from the fingertips of patients. The signal recorded was processed through an algorithm combining RMSSD and ShE. They evaluated the algorithm in 76 patients before and after cardioversion, effectively using each patient as their own control, and reported a sensitivity of $96.2 \%$, specificity of $97.5 \%$, and accuracy of 96.8\%. Krivoshei et al [19] applied the same published algorithm as McManus et al to detect AF with 80 consecutive patients. They demonstrated that the algorithm reliably discriminated between normal sinus rhythm and AF based on pulse wave signals from an iPhone $4 \mathrm{~S}$ camera only and achieved a sensitivity and specificity of $80 \%$ and $95 \%$, respectively. Rozen et al [26] conducted a study to assess the Cardiio Rhythm mobile phone app as a diagnostic tool in 97 patients before and after electrical cardioversion, and achieved a sensitivity of $93.1 \%$, a specificity of $90.9 \%$, a positive predictive value of $92.2 \%$, and a negative predictive value of $92.0 \%$ for $\mathrm{AF}$ detection. Bumgarner et al [27] reported that the Kardia Band algorithm from AliveCor paired with an Apple Smartwatch accurately differentiated AF from sinus rhythm in 100 patients before and after cardioversion, and demonstrated $93 \%$ sensitivity and $84 \%$ specificity. Tison et al [28] demonstrated that smartwatch PPG coupled with a deep neural network can passively detect AF compared to standard 12-lead ECG among 51 sedentary participants undergoing cardioversion with a sensitivity of $98.0 \%$ and specificity of $90.2 \%$, but with some loss of sensitivity $(67.7 \%)$ and specificity $(67.6 \%)$ in 1617 ambulatory participants.

Compared with other algorithms reported in previous studies, the PRO AF PPG algorithm performed better for AF screening in different smart devices with generally higher sensitivity, specificity, positive predictive value, negative predictive value, and accuracy. The majority of false positives originated from pulse waveforms that were corrupted by finger movement artifacts that may have affected the detection algorithm [22]. In fact, AF detection with single-lead ECG and PPG technology both should avoid the interference caused by movement to improve the accuracy. In this case, a 12-lead ECG was less affected by movement artifacts and may overall compare favorably in terms of inherent technical limitations compared to PPG. Although the diagnostic sensitivity of the PRO AF PPG algorithm was numerically lower compared to that from McManus et al [25] (95.56\% vs $96.20 \%)$, this may be due to our different study design. In this study, we analyzed heart rhythm data from 108 consecutive inpatients, whereas McManus et al performed repeated measurements in the same individual patients before and after cardioversion. We consider our study design more reasonable and closer to the intended use of $\mathrm{AF}$ detection in a large-scale, high-risk population.

Although short-term pulse waveform recordings with mobile phones for AF screening are superior to single spot-checks in the clinic, they are vulnerable to misdiagnosis in many patients with paroxysmal AF. We attempted to partially address this limitation by using smart bands, which can be worn on the wrist for 24 to 48 hours or even longer to obtain long-term pulsatile PPG signals. Therefore, mobile devices, either mobile phones or smart bands, may provide at-risk patients with important tools for screening AF in a single shot or over a long duration, which may help facilitate the early detection and early management of asymptomatic AF before a devastating outcome such as ischemic stroke occurs.

\section{Strengths and Limitations}

This is the first study on AF screening technology in China, and it demonstrates an easy AF screening approach using an algorithm based on smart devices with PPG and shows optimal diagnostic accuracy for heart rhythm readings. The advantage of AF screening with mobile phones is that it does not require additional hardware because optical video monitoring of the fingertip with a camera provides an accurate pulsatile time series related to variability in heart rate signals, making it more accessible and appealing to patients.

Some limitations of this study need to be addressed. First, we only focused on discriminating between $\mathrm{AF}$ and sinus rhythm in this study. However, the effect of sinus arrhythmia and other forms of ectopics, such as premature atrial beats, premature ventricular beats, and atrial flutter, on the performance of the algorithm should be evaluated, as these are common in the general population and might be similar in appearance to AF. In addition, the algorithm is unable to detect atrial flutter with a fixed atrioventricular conduction proportion that may also confer some risk of stroke and that frequently accompanies AF. Single-lead ECG may be used to detect AF and other arrhythmias. In our next study, new algorithms will be further developed to identify and differentiate sinus arrhythmia and various forms of ectopics. Second, although we reported the feasibility of using pulsatile PPG signals acquired from mobile phones and smart bands to detect AF in a group of participants 
preselected for their heart rhythm status, the ability to diagnose or screen AF with ambulatory outpatients has not been adequately investigated. Third, in this study, the data was collected in a supine position which could acquire stable pulse waveform signals and achieve reliable detection. However, the supine position is not common for home screening and creates inherent "not real world" rest conditions; therefore, it overestimates the results. Fourth, when we collected pulse waveform signals, we used the index or middle finger, not the pinky, which could have some effect on the results. However, it has been confirmed in several studies that using other fingers to collect pulse wave signals can also achieve good results $[19,25,29]$. Finally, although this was a pilot study, the sample size was relatively small, and more extensive clinical studies will need to be performed in the future.

\section{Conclusions}

Atrial fibrillation can be detected and heart rhythms analyzed using the broadly accessible smart devices with PPG technology. It provides an accurate and easy method of discriminating AF form sinus rhythm and may be used to detect asymptomatic patients with AF. Although PPG technology can be used to detect AF with good performance, the final diagnosis of $\mathrm{AF}$ must still be based on ECG according to current guidelines. Further studies are needed to assess the efficacy of this approach in detecting, screening, and diagnosing AF early, which we are currently performing.

\section{Acknowledgments}

The authors thank Hao Wang and Xiang-Ming Shi, Chinese PLA General Hospital, who provided guidance concerning statistical analysis. This research project was funded by the Chinese PLA Healthcare Foundation (17BJ208) and National Natural Science Foundation of China (H2501). HUAWEI (Huawei Technologies Co, Ltd, Shenzhen, China) provided the mobile phones (Mate 9, Honor 7X) and smart bands (Band 2) for study purposes. Preventicus (Preventicus GmbH, Jena, Germany) provided the Heartbeats mobile phone app and the PRO AF PPG algorithm.

\section{Authors' Contributions}

YYF conducted data collection and analysis and wrote the initial draft of the manuscript. YTG and YTW were the supervisors of this project as well as joint senior authors. YGL, JL, WKC, and ZLS provided guidance concerning statistical analysis. All coauthors contributed revisions to the manuscript and approved the final manuscript.

\section{Conflicts of Interest}

None declared.

\section{Multimedia Appendix 1}

Baseline characteristics of participants.

[PDF File (Adobe PDF File), 228KB-Multimedia Appendix 1]

\section{Multimedia Appendix 2}

Detailed diagnostic performance of the PPG technology for AF screening in different smart devices.

[PDF File (Adobe PDF File), 91KB-Multimedia Appendix 2]

\section{Multimedia Appendix 3}

Data from the literature on atrial fibrillation detection with different technologies.

[PDF File (Adobe PDF File), 176KB-Multimedia Appendix 3]

\section{References}

1. Lip GYH, Tse HF, Lane DA. Atrial fibrillation. Lancet 2012 Feb 18;379(9816):648-661. [doi: 10.1016/S0140-6736(11)61514-6] [Medline: 22166900]

2. Chugh SS, Havmoeller R, Narayanan K, Singh D, Rienstra M, Benjamin EJ, et al. Worldwide epidemiology of atrial fibrillation: a Global Burden of Disease 2010 Study. Circulation 2014 Feb 25;129(8):837-847 [FREE Full text] [doi: 10.1161/CIRCULATIONAHA.113.005119] [Medline: 24345399]

3. Cerasuolo JO, Montero-Odasso M, Ibañez A, Doocy S, Lip GY, Sposato LA. Decision-making interventions to stop the global atrial fibrillation-related stroke tsunami. Int J Stroke 2017 Dec;12(3):222-228. [doi: 10.1177/1747493016687579] [Medline: 28381196]

4. Lip GY, Lane DA. Stroke prevention in atrial fibrillation: a systematic review. JAMA 2015 May 19;313(19):1950-1962. [doi: 10.1001/jama.2015.4369] [Medline: 25988464] 
5. Vlachos K, Letsas KP, Korantzopoulos P, Liu T, Georgopoulos S, Bakalakos A, et al. Prediction of atrial fibrillation development and progression: current perspectives. World J Cardiol 2016 Mar 26;8(3):267-276 [FREE Full text] [doi: 10.4330/wjc.v8.i3.267] [Medline: 27022458]

6. Sposato LA, Cipriano LE, Saposnik G, Ruíz VE, Riccio PM, Hachinski V. Diagnosis of atrial fibrillation after stroke and transient ischaemic attack: a systematic review and meta-analysis. Lancet Neurol 2015 Apr;14(4):377-387. [doi: 10.1016/S1474-4422(15)70027-X] [Medline: 25748102]

7. Thihalolipavan S, Morin DP. Atrial fibrillation and congestive heart failure. Heart Fail Clin 2014 Apr;10(2):305-318. [doi: 10.1016/j.hfc.2013.12.005] [Medline: 24656107]

8. Xiong Q, Proietti M, Senoo K, Lip GY. Asymptomatic versus symptomatic atrial fibrillation: a systematic review of age/gender differences and cardiovascular outcomes. Int J Cardiol 2015 Jul 15;191:172-177. [doi:

10.1016/j.ijcard.2015.05.011] [Medline: 25974193]

9. Potpara TS, Lip GY. Oral anticoagulant therapy in atrial fibrillation patients at high stroke and bleeding risk. Prog Cardiovasc Dis 2015;58(2):177-194. [doi: 10.1016/j.pcad.2015.07.003] [Medline: 26162958]

10. Kirchhof P, Benussi S, Kotecha D, Ahlsson A, Atar D, Casadei B, et al. 2016 ESC Guidelines for the Management of Atrial Fibrillation Developed in Collaboration With EACTS. Rev Esp Cardiol (Engl Ed) 2017 Jan;70(1):50. [doi: 10.1016/j.rec.2016.11.033] [Medline: 28038729]

11. Svennberg E, Engdahl J, Al-Khalili F, Friberg L, Frykman V, Rosenqvist M. Mass screening for untreated atrial fibrillation: the STROKESTOP study. Circulation 2015 Jun 23;131(25):2176-2184 [FREE Full text] [doi: 10.1161/CIRCULATIONAHA.114.014343] [Medline: 25910800]

12. Aronsson M, Svennberg E, Rosenqvist M, Engdahl J, Al-Khalili F, Friberg L, et al. Cost-effectiveness of mass screening for untreated atrial fibrillation using intermittent ECG recording. Europace 2015 Jul;17(7):1023-1029. [doi: 10.1093/europace/euv083] [Medline: 25868469]

13. Capucci A, Calcagnini G, Mattei E, Triventi M, Bartolini P, Biancalana G, et al. Daily distribution of atrial arrhythmic episodes in sick sinus syndrome patients: implications for atrial arrhythmia monitoring. Europace 2012 Aug;14(8):1117-1124. [doi: 10.1093/europace/eus038] [Medline: 22406397]

14. Guo Y, Tian Y, Wang H, Si Q, Wang Y, Lip GY. Prevalence, incidence, and lifetime risk of atrial fibrillation in China: new insights into the global burden of atrial fibrillation. Chest 2015 Jan;147(1):109-119. [doi: 10.1378/chest.14-0321] [Medline: 24921459]

15. Bai Y, Wang Y, Shantsila A, Lip GY. The global burden of atrial fibrillation and stroke: a systematic review of the clinical epidemiology of atrial fibrillation in Asia. Chest 2017 Dec;152(4):810-820. [doi: 10.1016/j.chest.2017.03.048] [Medline: 28427968]

16. Verberk WJ, de Leeuw PW. Accuracy of oscillometric blood pressure monitors for the detection of atrial fibrillation: a systematic review. Expert Rev Med Devices 2012 Nov;9(6):635-640. [doi: 10.1586/erd.12.46] [Medline: 23249156]

17. Garabelli P, Stavrakis S, Po S. Smartphone-based arrhythmia monitoring. Curr Opin Cardiol 2017 Jan;32(1):53-57. [doi: 10.1097/HCO.0000000000000350] [Medline: 27875477]

18. Islam MS, Ammour N, Alajlan N, Aboalsamh H. Rhythm-based heartbeat duration normalization for atrial fibrillation detection. Comput Biol Med 2016 May 01;72:160-169. [doi: 10.1016/j.compbiomed.2016.03.015] [Medline: 27043858]

19. Krivoshei L, Weber S, Burkard T, Maseli A, Brasier N, Kühne M, et al. Smart detection of atrial fibrillation. Europace 2017 May 01;19(5):753-757 [FREE Full text] [doi: 10.1093/europace/euw125] [Medline: 27371660]

20. Taggar JS, Coleman T, Lewis S, Heneghan C, Jones M. Accuracy of methods for detecting an irregular pulse and suspected atrial fibrillation: a systematic review and meta-analysis. Eur J Prev Cardiol 2016 Dec;23(12):1330-1338 [FREE Full text] [doi: 10.1177/2047487315611347] [Medline: 26464292]

21. Kotecha D, Breithardt G, Camm AJ, Lip GY, Schotten U, Ahlsson A, et al. Integrating new approaches to atrial fibrillation management: the 6th AFNET/EHRA Consensus Conference. Europace 2018 Dec 01;20(3):395-407. [doi: 10.1093/europace/eux318] [Medline: 29300976]

22. Chan P, Wong C, Poh YC, Pun L, Leung WW, Wong Y, et al. Diagnostic performance of a smartphone-based photoplethysmographic application for atrial fibrillation screening in a primary care setting. J Am Heart Assoc 2016 Dec 21;5(7) [FREE Full text] [doi: 10.1161/JAHA.116.003428] [Medline: 27444506]

23. Spierer DK, Rosen Z, Litman LL, Fujii K. Validation of photoplethysmography as a method to detect heart rate during rest and exercise. J Med Eng Technol 2015;39(5):264-271. [doi: 10.3109/03091902.2015.1047536] [Medline: 26112379]

24. Carpenter A, Frontera A. Smart-watches: a potential challenger to the implantable loop recorder? Europace 2016 Jun;18(6):791-793. [doi: 10.1093/europace/euv427] [Medline: 26847074]

25. McManus DD, Lee J, Maitas O, Esa N, Pidikiti R, Carlucci A, et al. A novel application for the detection of an irregular pulse using an iPhone 4S in patients with atrial fibrillation. Heart Rhythm 2013 Mar;10(3):315-319 [FREE Full text] [doi: 10.1016/j.hrthm.2012.12.001] [Medline: 23220686]

26. Rozen G, Vaid J, Hosseini SM, Kaadan MI, Rafael A, Roka A, et al. Diagnostic accuracy of a novel mobile phone application for the detection and monitoring of atrial fibrillation. Am J Cardiol 2018 May 15;121(10):1187-1191. [doi:

10.1016/j.amjcard.2018.01.035] [Medline: 29525063] 
27. Bumgarner JM, Lambert CT, Hussein AA, Cantillon DJ, Baranowski B, Wolski K, et al. Smartwatch algorithm for automated detection of atrial fibrillation. J Am Coll Cardiol 2018 May 29;71(21):2381-2388. [doi: 10.1016/j.jacc.2018.03.003] [Medline: 29535065]

28. Tison GH, Sanchez JM, Ballinger B, Singh A, Olgin JE, Pletcher MJ, et al. Passive detection of atrial fibrillation using a commercially available smartwatch. JAMA Cardiol 2018 May 01;3(5):409-416. [doi: 10.1001/jamacardio.2018.0136] [Medline: 29562087]

29. Soni A, Karna S, Patel H, Fahey N, Raithatha S, Handorf A, et al. Study protocol for martphone onitoring for trial fibrillation in eal-ime in India (SMART-India): a community-based screening and referral programme. BMJ Open 2017 Dec 14;7(12):e017668 [FREE Full text] [doi: 10.1136/bmjopen-2017-017668] [Medline: 29247089]

\author{
Abbreviations \\ ACEI: angiotensin-converting-enzyme inhibitor \\ AF: atrial fibrillation \\ ARB: angiotensin receptor blockers \\ vascular disease, age 65-74, female sex \\ COPD: chronic obstructive pulmonary disease \\ ECG: electrocardiogram \\ FP: false positives \\ FN: false negatives \\ $>65$ years, drugs or alcohol \\ IQR: interquartile range \\ NPV: negative predictive value \\ PPA: Poincaré plot analysis \\ PPG: photoplethysmography \\ PPV: positive predictive value \\ RMSSD: root mean square of successive difference of RR intervals \\ SE: systemic arterial embolism \\ ShE: Shannon entropy \\ TIA: transient ischemic attack \\ TP: true positives \\ TN: true negatives
}

$\mathbf{C H A}_{2} \mathbf{D S}_{2}$-VASc: congestive heart failure, hypertension, age $\geq 75$ years, diabetes mellitus, stroke (doubled),

HAS-BLED: hypertension, abnormal renal function, abnormal liver function, stroke, bleeding, labile INR, age

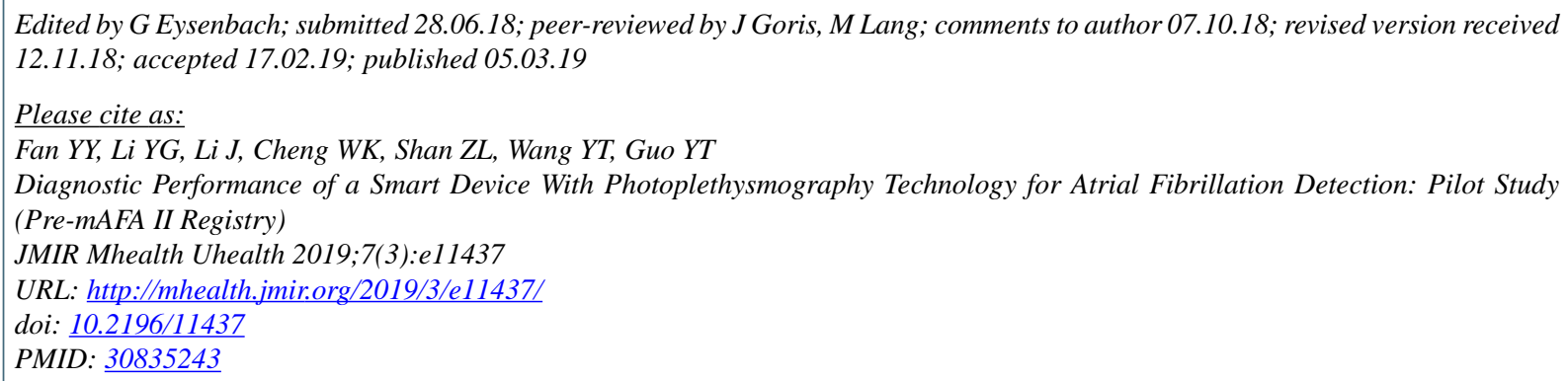

(C)Yong-Yan Fan, Yan-Guang Li, Jian Li, Wen-Kun Cheng, Zhao-Liang Shan, Yu-Tang Wang, Yu-Tao Guo. Originally published in JMIR Mhealth and Uhealth (http://mhealth.jmir.org), 05.03.2019. This is an open-access article distributed under the terms of the Creative Commons Attribution License (https://creativecommons.org/licenses/by/4.0/), which permits unrestricted use, distribution, and reproduction in any medium, provided the original work, first published in JMIR mhealth and uhealth, is properly cited. The complete bibliographic information, a link to the original publication on http://mhealth.jmir.org/, as well as this copyright and license information must be included. 